\title{
Epoxy Resin Embedding in Gelatin Capsules: Improved Orientation of Thin Specimens
}

\author{
Régis Pépin \\ Départment de Biologie Végétale, Bâtiment 405, \\ Université Claude Bernard (Lyon I), 43 Boulevard du 11 Novembre 1918, \\ 69621 Villeurbanne, France
}

Received June 3, 1975

When embedding samples destined to electron microscopical study, orientation is absolutely necessary in every case when some heterogeneity, in the structure of the specimen, requires that it should be studied, in sections, according to a determinated plane. The molds generally used for embedment-which are, most of the time, gelatin capsules ("gelules") for pharmaceutical use-do not allow an accurate orientation of the embedded pieces.

The methods generally adopted, i.e. double embedment or utilization of flat embedding molds, involve some inconvenients which limit their use. It is possible in certain cases to cut out the fragments to be included to such a shape that they can only rest, in the capsule, on those of their faces which are parallel to the desired sectionning plane. The shape itself of the capsules makes such a practice venturous. We are here suggesting a simple processing which can help to resolve some of the problems of the orientation.

\section{Material and method}

One can make up, in a very simple way, flat bottomed molds which allow, in only one time, embedment and orientation of the sample in most cases. It is easy indeed to modify the usual shape of embedding capsules. It is sufficient, for that, by hydrating it moderately, to soften locally the gelatin of which they are made. The water-treated area becomes malleable and then can be re-modeled. We took profit of this property to obtain flat bottomed embedding capsules, which are not industrially produced, to the best of our knowledge.

Practically, ordinary gelatin capsules, with their cap removed, are driven by force into holes of slightly smaller diameter, formerly pierced through a sheet of light cardboard, (Fig. 1) as it is often done to maintain the capsules vertical during the polymerisation of the embedding medium. One must take care that every capsule is about equally stuck into the holes, that will allow to moisten them all up to the same level. Anyway, one has interest, for that, not to treat more than 5 or 6 capsules at the same time.

The device thus realized is deposited in a jar (Petri dish or crystallizer) the bottom of which has been covered with a layer of cold $\left(15\right.$ to $\left.20^{\circ} \mathrm{C}\right)$ tap water, 4 to $5 \mathrm{~mm}$ thick (Fig. 1). The deepth of water to be used is critical, as it is necessary, for an easy subsequent handling, that the only part of the capsules the shape of 
which is to be changed should be hydrated. The precautions to be taken are limited to verify that the water layer has a constant deepth: so, one must insure oneself about the horizontality of the table or support, and the flatness of the jar bottom. We found plastic Petri dishes fully satisfactory on the later point.
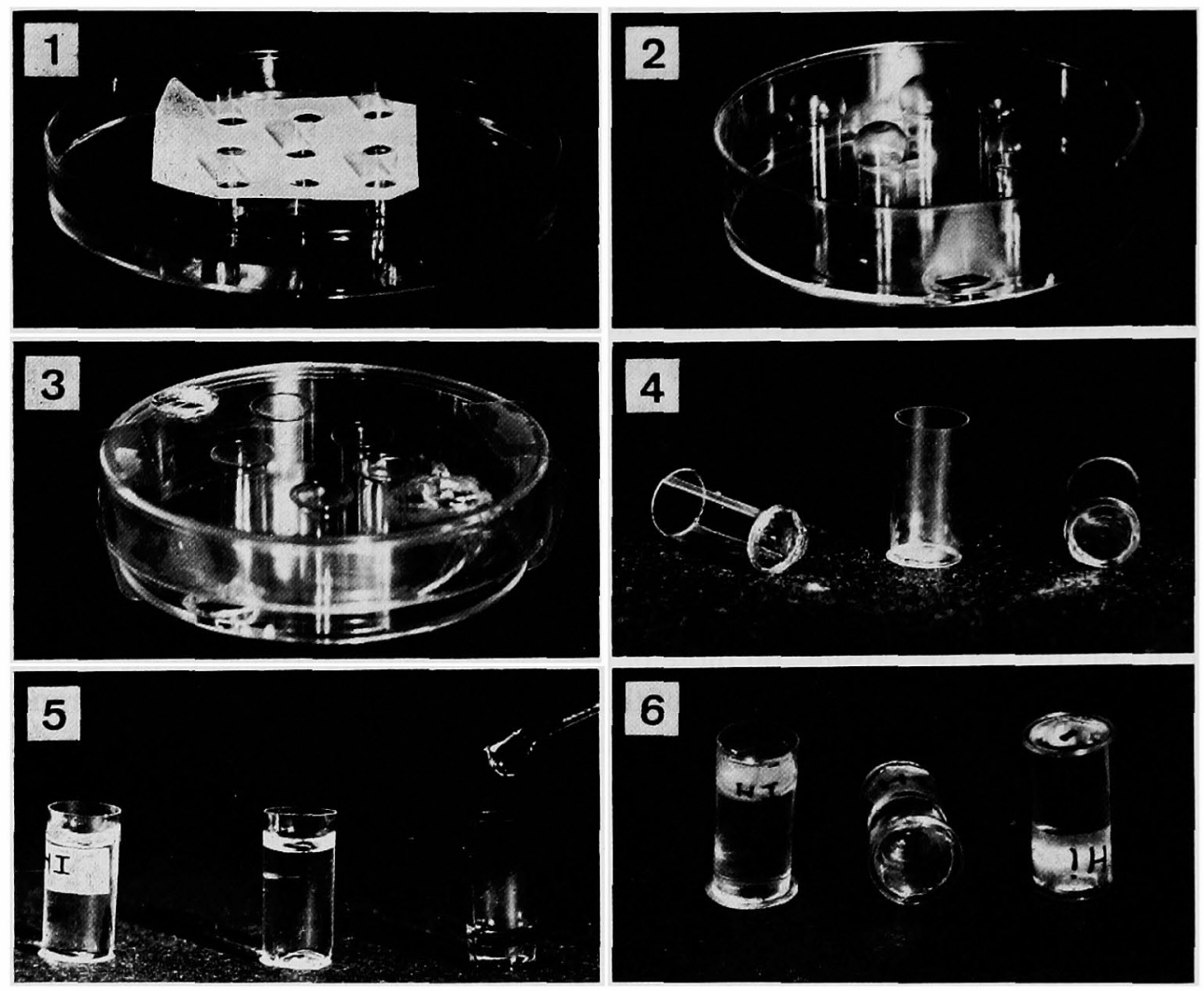

Figs. 1-6. 1, device for the hydratation of the capsules bottom, using a light cardboard holding sheet and a $10 \mathrm{~cm}$ in d. Petri dish, with a layer of tap water. 2, upside down disposition of moistened capsules, on the lower part of a $5 \mathrm{~cm}$ in d. Petri dish, showing their bottom slightly dilatated by water. 3 , pressing device using the upper part of the same Petri dish, (with holes pierced through) and adhesive tape to maintain the pressure. 4, several aspects of dried, achieved, flat bottomed capsules (storage form). 5, some stages of filling up flat bottomed capsules (self standing), with Mollenhauer's epon-araldite mixture, using a Pasteur pipette. 6, achieved polymerized inclusions of Sclerotinia tuberosa hymenium, showing the sample, which is orientated to produce longitudinal sections in the asci.

The extremity of the capsules is allowed to soak in water for five minutes. After this time, the device is taken off from the bath, and the capsules are quickly drained by gently shaking the whole assembly.

Handling them only with flat forceps, the capsules are removed from the sheet of cardboard, and disposed upside down, resting on their opening, hydrated part up, in the lower part of a polystyrene Petri dish (Fig. 2). The deepth of the dish must be adjusted in order that the hydrated area should exceed the height of its edge of 3 or $4 \mathrm{~mm}$ (i.e. about the length of the radius of the terminal hemispherical cap). 
5 or 6 capsules are thus disposed and, preferably after waiting 3 minutes to allow a slight beginning of drying, the top part of the Petri dish is set down onto the lower one, pressing and flattening the bottom of the capsules. As wet gelatin may be a little springy, pressure will be better maintened using two pieces of adhesive tape (Fig. 3).

One has interest to buy directly in the commerce Petri dishes of desired height. Could they not be found, higher dishes must be adjusted to the required height by abrasion on a sheet of emery-cloth. In this case, the horizontality of the cover has to be verified with a level, and corrected, if necessary, so as to be perfect.

In all cases, wide enough holes (about $5 \mathrm{~mm}$ in d.) must be pierced through the peripheral region of both cover and bottom of the Petri dish (Fig. 3) to allow the water to evaporate faster. Since the use of a vacuum dryer should be avoided, drying the capsules will be done in air, by placing the whole disposal-we found upside down better-in an oven at $40^{\circ} \mathrm{C}$.

The modified Petri dishes utilized here can be used as many times as one wants: after having rehardened when getting dry, the modified capsules will come away, by themselves, from the cover which was used to flatten them, without any damage for it. The obtained capsules (Fig. 4) can be then stored indefinitely, in a dry place, after that those which have not the exactly desired shape have been eliminated. The method is of good efficiency: most of the time, 8 or 9 capsules, on ten treated, can be utilized.

The capsules will be dried up one more time, by placing them for an hour at $40^{\circ} \mathrm{C}$ just before they are used. Our embedments were made with Mollenhauer's (1964) epon-araldite embedding mixture (Fig. 5), but it seems that any other embedding medium might be found as well convenient.

\section{Applications and discussion}

We took profit of this technique to cut out longitudinal sections in hymenial asci and paraphysae of the inoperculate Discomycete fungus Sclerotinia tuberosa (Fig. 6). But we mainly applied it as a simplification of the sectionning technique that we had formerly developped: Pepin (1974), for the electron microscopical study of the fungal mycelia in the plane of the filaments. It seems that it can be applied, in the same way, to any of the others embedding methods concerning flat specimens: Langvad (1971), for exemple. Especially in these cases, rubber molds have many inconvenients: plus their expensiveness and rapid wearing, they are made such in a way that the sample must rest on the bottom of the lodges. When embedding large, cube-shaped or prismatic samples, they can be very helpful. But acting of thin specimens such as fungal mycelium or cells monolayers, they require either little safe sticking or double embedment, with particuliar precautions to be taken, to obtain a well appropriate polymerisation.

One has therefore interest to replace the flat embedding molds by flat bottomed capsules which allow, at the contrary, the simultaneous embedment and orientation of this kind of specimen. The resulting very accurate orientation even avoids to have recourse to corrective processes such as Hwang's (1970). The incorporated 
adjustment devices of the microtomes are enough to rectify the slight defects in orientation which may exist in the achieved embedment.

Let us mention that setting up this kind of embedded sample on microtomes does not necessitate, as for flat-embedded ones, any special sample-holder.

Although they seem mainly useful in the cases that we have mentionned, the modified capsules can be used systematically, in place of flat embedding molds. They present, in any case the advantage of producing properly centered embedments. However, flat molds will remain the only possible solution when one wants to cut out sections from a thin sample, perpendicularly to the plane of flatness.

In the almost general case where it can be used to orientate the specimens, the technique that we are describing allows to choose as a sectionning surface every part of them that is in contact with the flattened bottom of the capsule. This is not always possible with orientated embedments using ordinary capsules.

\section{Conclusion}

The utilization of flat bottomed capsules seems to present some positive advantages on the usual embedding processes for ultramicrotomy. The simpleness of this technique and the subsequent reliability of its results, the facility of obtaining the required material, its absolute unexpensiveness, allow its widespread use in most cases when orientated embedments, for electron microscope study, are wished for, avoiding too long manipulations.

\section{Abstract}

The hemispherical bottom of ordinary gelatin capsules can be softened by partial soaking in cold water. Pressing the moistened part with a smooth, flatsurfaced object will modify the original shape of the capsule to a flat-bottomed one. Plastic Petri dishes can be used for this work with excellent results. This new shape becomes stable after drying and the capsules thus obtained can be used for orientated embedment of most kinds of samples. Using these modified capsules is particularly helpful for embedding thin specimens such as cells monolayers or fungal mycelia.

\section{References}

Hwang, Y. C. 1970. A modification for orientation by the use of silicone rubber molds for embedding tissues for electron microscopy. J. Electron Microsc. 19 (2): 189-190.

Langvad, F. 1971. Embedding of filamentous fungi for electron microscopy. Laboratory Practice. 20 (4): 335.

Mollenhauer, H. H. 1964. Plastic embedding mixtures for electron microscopy. Stain Technol. $39(2): 111-114$.

Pépin, R. 1974. Une technique d'obtention de coupes ultrafines longitudinales dans les hyphes mycéliennes des champignons, pour la microscopie électronique. Mycopathol. and Mycol. appl. 52 (2): 85-91. 